\title{
New Method of Solvability of a Three-dimensional Laplace Equation with Nonlocal Boundary Conditions
}

\author{
Y.Y. Mustafayeva and N.A. Aliyev \\ Baku State University \\ 23 Z. Khalilov Str., AZ 1148, Baku, Azerbaijan \\ E-mail: helenmust@rambler.ru \\ aliyev.nihan@mail.ru
}

Received November 9, 2014, revised October 23, 2015

\begin{abstract}
The solutions of a boundary problem with non-local boundary conditions for a three-dimensional Laplace equation are studied. Here, the boundary conditions are the most common and linear. Further, we note that the singular integrals appearing in the necessary conditions are multi-dimensional. Therefore, the regularization of these singularities is much more difficult than the regularization of one-dimensional singular integrals. After the regularization of singularities the Fredholm property of the problem is proved.

Key words: non-local boundary conditions, three-dimensional Laplace equation, multi-dimensional singular integral, necessary conditions, regularization, Fredholm property.
\end{abstract}

Mathematics Subject Classification 2010: 35J05, 35J40.

\section{Introduction}

We have been studying boundary value problems and the related conditions since the 1970s. After publishing the results on ODE's, we began to study the problems for partial differential equations. The first published work in this direction was the problem for an elliptic equation of first order, i.e., the CauchyRiemann equation [1], and the second was the problem for the Cauchy-Riemann equation with nonlocal boundary conditions [2]. Note that if we consider the Cauchy-Riemann equation with local boundary conditions (Dirichlet condition), this problem is incorrect. Further we considered the Cauchy-Riemann equation with non-local and global terms in the boundary conditions [3]. The extension of that work on an integral-differential equation with the Cauchy-Riemann equation as its principal part and non-local conditions of the general form was given in 
[4]. A mixed-type equation of the first order with nonlocal boundary conditions was considered in [5], and a Steklov problem for the Cauchy-Riemann equation was considered in [6]. The inverse problem for the Cauchy-Riemann equation in the Tikhonov-Lavrentiev sense was considered in [7]. Also there was studied the effect of Carleman conditions on the Fredholm property of a problem for the Cauchy-Riemann equation [8].

The results obtained for the Cauchy-Riemann equation were extended to a boundary-value problem for the two-dimensional Laplace equation in [9], [10] and the Steklov problem for this equation, in [11]. The methods proposed and used in the above works were applied to the study of solutions to the mixed problem for the equations of parabolic and hyperbolic types [12-15].

The boundary value problem for a two-dimensional integro-differential loaded equation with boundary conditions containing both nonlocal and global terms (integrals) was considered in [16], and boundary-value problems for an equation of composite type with general linear nonlocal boundary conditions were studied by this method in $[17,18]$. A mixed problem for the Navier-Stokes equations was considered in $[19,20]$. The present work deals with the solutions to the boundary value problem for the three-dimensional Laplace equation with nonlocal boundary conditions. The analyses of boundary value problems for the Laplace equation with nonlocal boundary conditions were conducted by a different method in [21].

A version of the new method of [21] was applied in [11] to the two-dimensional Laplace equation. However, for the three-dimensional case the reasoning is more complicated, and this is for the first time that we give a complete proof, in particular, that of the Fredholm property.

The difference of the new method from the previous ones (e.g., the method of successive approximations [22, p. 74], [23]) applied to a one- and a twodimensional equations [24] is as follows. In our case the singular integral equations in the obtained necessary conditions are in the spectrum, i.e., if one applies only one iteration, we obtain a singular integral equation with the same singularity. If one applies the Poincare-Bertrand formula, the resulting jump will be eliminated by the external term. As a result, the Fredholm integral equation of the first kind is obtained.

The approach is based on the necessary conditions for the existence of the solution to the problem which are obtained in the work. Note that the necessary conditions for a linear ODE are obtained in the form of conventional boundary conditions.

If $\mathrm{n}$ necessary conditions are obtained for an $n$-th order differential equation with $\mathrm{n}$ boundary conditions, then we obtain a system of $2 n$ linear algebraic equations (the unknowns are the boundary values of the unknown function and its derivatives up to the ( $n-1)$-th order inclusive). If we can determine the unknowns from this system, then the solution of the problem is obtained from the Lagrange 
formula. If the resulting system of linear algebraic equations is unsolvable, then the boundary value problem has no solution either. If the system has an infinite number of solutions, then the boundary value problem has an infinite number of solutions too (if at least one boundary condition is a linear combination of the necessary conditions or vice versa).

These necessary conditions are derived from the basic equation of the problem and the fundamental solution of the adjoint equation using the second Green's formula (it suffices to use the Lagrange formula for an ODE).

Some necessary conditions (for partial differential equations) contain singularities. The regularization of singular integrals cannot be obtained by usual methods. That is why, in this paper, we present the new schemes of regularization. We also derive below an analogue of the second Green's formula for derivatives and use it to obtain a complete system of necessary conditions.

Combining the regularized necessary conditions with the given boundary conditions, we obtain sufficient conditions for the Fredholm property of the problem.

\section{Problem Statement}

Let us consider the three-dimensional Laplace equation in the domain $D \subset R^{3}$, convex in the direction $x_{3}$, whose projection onto the plane $O x_{1} x_{2}=O x^{\prime}$ is the domain $S \subset O x_{1} x_{2}, \Gamma$ is the boundary (surface) of the domain $D$ :

$$
\begin{gathered}
L u=\Delta u(x)=\frac{\partial^{2} u(x)}{\partial x_{1}^{2}}+\frac{\partial^{2} u(x)}{\partial x_{2}^{2}}+\frac{\partial^{2} u(x)}{\partial x_{3}^{2}}=0, \\
x=\left(x_{1}, x_{2}, x_{3}\right) \in D,
\end{gathered}
$$

with non-local boundary conditions:

$$
\begin{gathered}
l_{i} u=\sum_{j=1}^{3}\left[\left.\alpha_{i j}^{(1)}\left(x^{\prime}\right) \frac{\partial u(x)}{\partial x_{j}}\right|_{x_{3}=\gamma_{1}\left(x^{\prime}\right)}+\left.\alpha_{i j}^{(2)}\left(x^{\prime}\right) \frac{\partial u(x)}{\partial x_{j}}\right|_{x_{3}=\gamma_{2}\left(x^{\prime}\right)}\right] \\
+\alpha_{i}^{(1)}\left(x^{\prime}\right) u\left(x^{\prime}, \gamma_{1}\left(x^{\prime}\right)\right)+\alpha_{i}^{(2)}\left(x^{\prime}\right) u\left(x^{\prime}, \gamma_{2}\left(x^{\prime}\right)\right)=f_{i}\left(x^{\prime}\right), \\
i=1,2 ; x^{\prime} \in S, \\
u(x)=f_{0}(x), x \in \bar{\Gamma}_{1} \bigcap \bar{\Gamma}_{2} .
\end{gathered}
$$

Here $\Gamma_{1}$ and $\Gamma_{2}$ are the upper and lower half-surfaces of the boundary $\Gamma$, respectively, defined as $\Gamma_{k}=\left\{\xi=\left(\xi_{1}, \xi_{2}, \xi_{3}\right): \xi_{3}=\gamma_{k}\left(\xi^{\prime}\right), \xi^{\prime}=\left(\xi_{1}, \xi_{2}\right) \in S=\operatorname{pr}_{\xi_{3}=0} \Gamma_{k}\right\}$, $k=1,2$, where $\xi_{3}=\gamma_{k}\left(\xi_{1}, \xi_{2}\right), k=1,2$, are equations of the half-surfaces $\Gamma_{1}$ and $\Gamma_{2}$; the functions $\gamma_{k}\left(\xi^{\prime}\right), k=1,2$, are twice differentiable with respect to 
the variables $\left(\xi_{1}, \xi_{2}\right)$; the coefficients $\alpha_{i j}^{(k)}\left(x^{\prime}\right), \alpha_{i}^{(k)}\left(x^{\prime}\right), k=1,2$, are continuous functions in the domain $S$.

The fundamental solution for the three-dimensional Laplace equation has the form of $[25]$ :

$$
U(x-\xi)=-\frac{1}{4 \pi} \frac{1}{|x-\xi|}
$$

\section{Basic Relationships and Necessary Conditions}

Multiplying Eq. (2.1) by the fundamental solution (2.4), integrating it over the domain $D$ and taking into account that $\Delta_{x} U(x-\xi)=\delta(x-\xi)$, where $\delta(x-\xi)$ is the Dirac $\delta$-function, we can get the first basic relationship:

$$
\begin{gathered}
-\sum_{j=1}^{3} \int_{\Gamma}\left[\left(\frac{\partial u(x)}{\partial x_{j}} U(x-\xi)-u(x) \frac{\partial U(x-\xi}{\partial x_{j}}\right) \cos \left(\nu, x_{j}\right) d x\right] \\
=\int_{D} u(x) \delta(x-\xi) d x= \begin{cases}u(\xi), & \xi \in D, \\
\frac{1}{2} u(\xi), & \xi \in \Gamma .\end{cases}
\end{gathered}
$$

Here the first relationship gives the representation of the general solution of equation (2.1), and the second expression in (3.1) is the first necessary condition.

Consider the first necessary condition $(\xi \in \Gamma)$ :

$$
\begin{aligned}
& \frac{1}{2} u(\xi)=-\sum_{j=1}^{3} \int_{\Gamma}\left(\frac{\partial u(x)}{\partial x_{j}} U(x-\xi)-u(x) \frac{\partial U(x-\xi)}{\partial x_{j}}\right) \cos \left(\nu_{x}, x_{j}\right) d x \\
& =-\int_{\Gamma} \frac{\partial u(x)}{\partial \nu} U(x-\xi) d x+\int_{\Gamma} u(x) \sum_{j=1}^{3} \frac{\partial U(x-\xi)}{\partial x_{j}} \cos \left(\nu_{x}, x_{j}\right) d x .
\end{aligned}
$$

As $\frac{\partial U(x-\xi)}{\partial x_{i}}=-\frac{x_{i}-\xi_{i}}{4 \pi|x-\xi|^{3}}=-\frac{\cos \left(x-\xi, x_{i}\right)}{4 \pi|x-\xi|^{2}}$, all the integrands in (3.2) have a weak singularity, e.i., the order of singularity does not exceed the multiplicity of integrals.

Thus we have proved

Theorem 3.1. Let $D \subset R^{3}$ be a bounded domain, convex in the $x_{3}$-direction and such that its boundary $\Gamma$ is a Lyapunov surface. Then the obtained first necessary condition (3.2) is regular. 
Multiplying (2.1) by $\frac{\partial U(x-\xi)}{\partial x_{i}}, i=\overline{1,3}$, and integrating it over the domain $D$, we obtain the rest of three basic relationships:

$$
\begin{gathered}
\int_{\Gamma} \frac{\partial u(x)}{\partial x_{i}} \frac{\partial U(x-\xi)}{\partial \nu_{x}} d x \\
+\int_{\Gamma} \frac{\partial u(x)}{\partial x_{m}}\left[\frac{\partial U(x-\xi)}{\partial x_{i}} \cos \left(\nu_{x}, x_{m}\right)-\frac{\partial U(x-\xi)}{\partial x_{m}} \cos \left(\nu_{x}, x_{i}\right)\right] d x \\
+\int_{\Gamma} \frac{\partial u(x)}{\partial x_{l}}\left[\frac{\partial U(x-\xi)}{\partial x_{i}} \cos \left(\nu_{x}, x_{l}\right)-\frac{\partial U(x-\xi)}{\partial x_{l}} \cos \left(\nu_{x}, x_{i}\right)\right] d x \\
= \begin{cases}-\frac{\partial u(\xi)}{\partial \xi_{i}}, & \xi \in D, \\
-\frac{1}{2} \frac{\partial u(\xi)}{\partial \xi_{i}}, & \xi \in \Gamma,\end{cases}
\end{gathered}
$$

where the triple $i, m, l$ is a permutation of numbers $1,2,3$.

The second expressions in (3.3) are the other three necessary conditions $(\xi \in \Gamma, i=1,3)$ :

$$
\begin{gathered}
-\frac{1}{2} \frac{\partial u(\xi)}{\partial \xi_{i}} \\
=\int_{\Gamma} \frac{\partial u(x)}{\partial x_{i}} \frac{\partial U(x-\xi)}{\partial \nu_{x}} d x \\
+\int_{\Gamma} \frac{\partial u(x)}{\partial x_{m}}\left[\frac{\partial U(x-\xi)}{\partial x_{i}} \cos \left(\nu_{x}, x_{m}\right)-\frac{\partial U(x-\xi)}{\partial x_{m}} \cos \left(\nu_{x}, x_{i}\right)\right] d x \\
+\int_{\Gamma} \frac{\partial u(x)}{\partial x_{l}}\left[\frac{\partial U(x-\xi)}{\partial x_{i}} \cos \left(\nu_{x}, x_{l}\right)-\frac{\partial U(x-\xi)}{\partial x_{l}} \cos \left(\nu_{x}, x_{i}\right)\right] d x .
\end{gathered}
$$

where the triple $i, m, l$ is a permutation of numbers $1,2,3$.

Taking into account that $\frac{\partial U(x-\xi)}{\partial x_{i}}=-\frac{x_{i}-\xi_{i}}{4 \pi|x-\xi|^{3}}=-\frac{\cos \left(x-\xi, x_{i}\right)}{4 \pi|x-\xi|^{2}}$ and introducing the designations

$$
K_{i j}(x, \xi)=\left(\cos \left(x-\xi, x_{i}\right) \cos \left(\nu_{x}, x_{j}\right)-\cos \left(x-\xi, x_{j}\right) \cos \left(\nu_{x}, x_{i}\right)\right),
$$

we can write the 2 nd, the 3 rd and the 4 th necessary conditions (3.4) in the form: 


$$
\begin{gathered}
-\frac{1}{2} \frac{\partial u(\xi)}{\partial \xi_{i}} \\
=\int_{\Gamma} \frac{\partial u(x)}{\partial x_{i}} \frac{\partial U(x-\xi)}{\partial \nu_{x}} d x \\
+\int_{\Gamma} \frac{\partial u(x)}{\partial x_{m}} \frac{K_{i m}(x, \xi)}{4 \pi|x-\xi|^{2}} d x+\int_{\Gamma} \frac{\partial u(x)}{\partial x_{l}} \frac{K_{i l}(x, \xi)}{4 \pi|x-\xi|^{2}} d x,
\end{gathered}
$$

where the triple $i, m, l$ is a permutation of numbers $1,2,3$.

To introduce the second group of necessary conditions we write the two first surface integrals in the $(i+1)$-th relationship $(3.6)(i=1,2,3)$ over the upper and lower half-surfaces $\Gamma_{k}, k=1,2$ in the form:

$$
\begin{aligned}
& -\left.\frac{1}{2} \frac{\partial u}{\partial \xi_{i}}\right|_{\xi_{3}=\gamma_{k}\left(\xi^{\prime}\right)} \\
& =\left.\sum_{j=1}^{2}(-1)^{j-1} \int_{S} \frac{\partial u(x)}{\partial x_{m}}\right|_{x_{3}=\gamma_{j}\left(x^{\prime}\right)} \frac{K_{i m}(x, \xi)}{4 \pi|x-\xi|^{2}} \mid \begin{array}{l}
x_{3}=\gamma_{j}\left(x^{\prime}\right) \\
\begin{array}{l}
\cos \left(\nu_{x}, x_{3}\right) \\
\xi_{3}=\gamma_{k}\left(\xi^{\prime}\right)
\end{array}
\end{array} \\
& +\left.\sum_{j=1}^{2}(-1)^{j-1} \int_{S} \frac{\partial u(x)}{\partial x_{l}}\right|_{x_{3}=\gamma_{j}\left(x^{\prime}\right)} \frac{K_{i l}(x, \xi)}{4 \pi|x-\xi|^{2}} \mid \begin{array}{l}
x_{3}=\gamma_{j}\left(x^{\prime}\right) \\
\begin{array}{l}
\cos \left(\nu_{x}, x_{3}\right) \\
\xi_{3}=\gamma_{k}\left(\xi^{\prime}\right)
\end{array}
\end{array} \\
& +\left.\int_{\Gamma} \frac{\partial u(x)}{\partial x_{i}} \frac{\partial U(x-\xi)}{\partial \nu_{x}}\right|_{\xi_{3}=\gamma_{k}\left(\xi^{\prime}\right)} d x .
\end{aligned}
$$

The singular terms for $=1,2$ in the 2 nd, 3rd, 4th necessary conditions $(i=$ $1,3)$ are:

$$
\begin{aligned}
& \left.\frac{1}{2} \frac{\partial u}{\partial \xi_{i}}\right|_{\xi_{3}=\gamma_{k}\left(\xi^{\prime}\right)}=\left.(-1)^{k} \int_{S} \frac{\partial u(x)}{\partial x_{m}}\right|_{x_{3}=\gamma_{k}\left(x^{\prime}\right)} \frac{K_{i m}(x, \xi)}{4 \pi|x-\xi|^{2}} \mid \begin{array}{l}
x_{3}=\gamma_{k}\left(x^{\prime}\right) \\
\begin{array}{l}
\cos \left(\nu_{x}, x_{3}\right) \\
\xi_{3}=\gamma_{k}\left(\xi^{\prime}\right)
\end{array}
\end{array}
\end{aligned}
$$

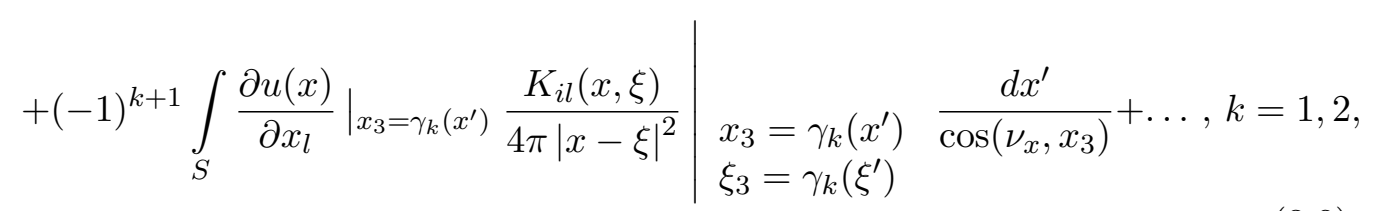


where three dots denote the sum of nonsingular terms.

$\mathrm{R}$ e $\mathrm{m}$ a $\mathrm{r} \mathrm{k}$ 3.1. Three dots in (3.8) contain the derivatives $\left.\frac{\partial u(x)}{\partial x_{i}}\right|_{x_{3}=\gamma_{k}\left(x^{\prime}\right)}$, $l=1,2,3 ; k=1,2$, under the sing of integral and we will take it into consideratiob later.

Let us introduce the designations:

$$
K_{i j}^{(k)}\left(x^{\prime}, \xi^{\prime}\right)=K_{i j}(x, \xi) \mid \begin{aligned}
& x_{3}=\gamma_{k}\left(x^{\prime}\right) \quad, \quad k=1,2 . \\
& \xi_{3}=\gamma_{k}\left(\xi^{\prime}\right)
\end{aligned}
$$

$$
\begin{aligned}
& \text { We consider }|x-\xi|^{2} \mid \begin{array}{l}
x_{3}=\gamma_{k}\left(x^{\prime}\right) \\
\xi_{3}=\gamma_{k}\left(\xi^{\prime}\right)
\end{array}, k=1,2 \text { : } \\
& |x-\xi|^{2}\left|\begin{array}{l}
x_{3}=\gamma_{k}\left(x^{\prime}\right) \\
\xi_{3}=\gamma_{k}\left(\xi^{\prime}\right)
\end{array}=\right| x^{\prime}-\left.\xi^{\prime}\right|^{2}+\left(\gamma_{k}\left(x^{\prime}\right)-\gamma_{k}\left(\xi^{\prime}\right)\right)^{2} \\
& =\left|x^{\prime}-\xi^{\prime}\right|^{2}\left[1+\sum_{m=1}^{2}\left(\frac{\partial \gamma_{k}\left(x^{\prime}\right)}{\partial x_{m}}\right)^{2} \cos ^{2}\left(x^{\prime}-\xi^{\prime}, x_{m}\right)+O\left(\left|x^{\prime}-\xi^{\prime}\right|\right)\right] .
\end{aligned}
$$

Let us introduce the designations:

$$
P_{k}\left(x^{\prime}, \xi^{\prime}\right)=1+\sum_{m=1}^{2}\left(\frac{\partial \gamma_{k}\left(x^{\prime}\right)}{\partial x_{m}}\right)^{2} \cos ^{2}\left(x^{\prime}-\xi^{\prime}, x_{m}\right)+O\left(\left|x^{\prime}-\xi^{\prime}\right|\right),
$$

whence we have that

$$
|x-\xi|^{2}\left|\begin{array}{l}
x_{3}=\gamma_{k}\left(x^{\prime}\right) \\
\xi_{3}=\gamma_{k}\left(\xi^{\prime}\right)
\end{array}=\right| x^{\prime}-\left.\xi^{\prime}\right|^{2} P_{k}\left(x^{\prime}, \xi^{\prime}\right) .
$$

$\mathrm{R}$ e $\mathrm{m}$ a r k 3.2 . Notice that for $\xi^{\prime}=x^{\prime}$ we have

$$
P_{k}\left(x^{\prime}, x^{\prime}\right)=1+\left(\frac{\partial \gamma_{k}}{\partial x_{1}}\right)^{2}+\left(\frac{\partial \gamma_{k}}{\partial x_{2}}\right)^{2}+2 \frac{\partial \gamma_{k}}{\partial x_{1}} \frac{\partial \gamma_{k}}{\partial x_{2}} \neq 0, k=1,2
$$

By means of designations (3.9), (3.10) we can rewrite the necessary conditions (3.8) for $k=1,2$ as follows: 


$$
\begin{gathered}
\left.\frac{1}{2} \frac{\partial u}{\partial \xi_{i}}\right|_{\xi_{3}=\gamma_{k}\left(\xi^{\prime}\right)} \\
=\left.(-1)^{(k)} \int_{S} \frac{\partial u(x)}{\partial x_{m}}\right|_{x_{3}=\gamma_{k}\left(x^{\prime}\right)} \frac{1}{4 \pi\left|x^{\prime}-\xi^{\prime}\right|^{2}} \frac{K_{i m}^{(k)}\left(x^{\prime}, \xi^{\prime}\right)}{P_{k}\left(x^{\prime}, \xi^{\prime}\right)} \frac{d x^{\prime}}{\cos \left(\nu_{x}, x_{3}\right)} \\
+\left.(-1)^{(k+1)} \int_{S} \frac{\partial u(x)}{\partial x_{l}}\right|_{x_{3}=\gamma_{k}\left(x^{\prime}\right)} \frac{1}{4 \pi\left|x^{\prime}-\xi\right|^{\prime 2}} \frac{K_{i l}^{(k)}\left(x^{\prime}, \xi^{\prime}\right)}{P_{k}\left(x^{\prime}, \xi^{\prime}\right)} \frac{d x^{\prime}}{\cos \left(\nu_{x}, x_{3}\right)}+\ldots \\
i=1,2,3 ; k=1,2 .
\end{gathered}
$$

Theorem 3.2. Under assumptions of Theorem 3.1 necessary conditions (3.11) are singular.

\section{Regularization of the Necessary Conditions}

Let us build a linear combination of necessary conditions (3.11) for $k=1,2$ $(j=1,2,3)$ :

$$
\begin{gathered}
\left.\beta_{i j}^{(1)}\left(\xi^{\prime}\right) \frac{\partial u(\xi)}{\partial \xi_{j}}\right|_{\xi_{3}=\gamma_{1}\left(\xi^{\prime}\right)}+\left.\beta_{i j}^{(2)}\left(\xi^{\prime}\right) \frac{\partial u(\xi)}{\partial \xi_{j}}\right|_{\xi_{3}=\gamma_{2}\left(\xi^{\prime}\right)} \\
=\int_{S} \sum_{k=1}^{2} \beta_{i j}^{(k)}\left(\xi^{\prime}\right)(-1)^{k}\left(\left.\frac{\partial u(x)}{\partial x_{m}}\right|_{x_{3}=\gamma_{k}\left(x^{\prime}\right)} \frac{K_{j m}^{(k)}(x, \xi)}{P_{k}\left(x^{\prime}, \xi^{\prime}\right)}+\left.\frac{\partial u(x)}{\partial x_{l}}\right|_{x_{3}=\gamma_{k}\left(x^{\prime}\right)} \frac{K_{j l}^{(k)}(x, \xi)}{P_{k}\left(x^{\prime}, \xi^{\prime}\right)}\right) \\
\times \frac{1}{2 \pi\left|x^{\prime}-\xi^{\prime}\right|^{2}} \frac{d x^{\prime}}{\cos \left(\nu_{x}, x_{3}\right)}+\ldots
\end{gathered}
$$

where the triple $j, m, l$ is a permutation of numbers $1,2,3$.

Form a sum of (4.1) for $j=1,2,3$ and factor out the common factor $\frac{1}{2 \pi\left|x^{\prime}-\xi^{\prime}\right|^{2}}$ under the sign of integral $(i=1,2)$ :

$$
\begin{gathered}
\sum_{j=1}^{3}\left(\left.\beta_{i j}^{(1)}\left(\xi^{\prime}\right) \frac{\partial u(\xi)}{\partial \xi_{j}}\right|_{\xi_{3}=\gamma_{1}\left(\xi^{\prime}\right)}+\left.\beta_{i j}^{(2)}\left(\xi^{\prime}\right) \frac{\partial u(\xi)}{\partial \xi_{j}}\right|_{\xi_{3}=\gamma_{2}\left(\xi^{\prime}\right)}\right) \\
=\int_{S} \frac{1}{2 \pi\left|x^{\prime}-\xi^{\prime}\right|^{2}} \frac{d x^{\prime}}{\cos \left(\nu_{x}, x_{3}\right)} \sum_{k=1}^{2}(-1)^{k} \sum_{j=1}^{3} \beta_{i j}^{(k)}\left(\xi^{\prime}\right)
\end{gathered}
$$




$$
\times\left(\left.\frac{\partial u(x)}{\partial x_{2}}\right|_{x_{3}=\gamma_{k}\left(x^{\prime}\right)} \frac{K_{j m}^{(k)}\left(x^{\prime}, \xi^{\prime}\right)}{P_{k}\left(x^{\prime}, \xi^{\prime}\right)}+\left.\frac{\partial u(x)}{\partial x_{3}}\right|_{x_{3}=\gamma_{k}\left(x^{\prime}\right)} \frac{K_{j l}^{(k)}\left(x^{\prime}, \xi^{\prime}\right)}{P_{k}\left(x^{\prime}, \xi^{\prime}\right)}\right)+\ldots
$$

Adding and subtracting $\beta_{i j}^{(k)}\left(x^{\prime}\right)$ to and from $\beta_{i j}^{(k)}\left(\xi^{\prime}\right), k=1,2$ in (4.2), we obtain

$$
\begin{gathered}
\sum_{j=1}^{3}\left(\left.\beta_{i j}^{(1)}\left(\xi^{\prime}\right) \frac{\partial u(\xi)}{\partial \xi_{j}}\right|_{\xi_{3}=\gamma_{1}\left(\xi^{\prime}\right)}+\left.\beta_{i j}^{(2)}\left(\xi^{\prime}\right) \frac{\partial u(\xi)}{\partial \xi_{j}}\right|_{\xi_{3}=\gamma_{2}\left(\xi^{\prime}\right)}\right) \\
=\int_{S} \frac{1}{2 \pi\left|x^{\prime}-\xi^{\prime}\right|^{2}} \frac{d x^{\prime}}{\cos \left(\nu_{x}, x_{3}\right)} \sum_{k=1}^{2}(-1)^{k} \sum_{j=1}^{3} \beta_{i j}^{(k)}\left(x^{\prime}\right) \\
\times\left(\left.\frac{\partial u(x)}{\partial x_{m}}\right|_{x_{3}=\gamma_{k}\left(x^{\prime}\right)} \frac{K_{j m}^{(k)}\left(x^{\prime}, \xi^{\prime}\right)}{P_{k}\left(x^{\prime}, \xi^{\prime}\right)}+\left.\frac{\partial u(x)}{\partial x_{l}}\right|_{x_{3}=\gamma_{k}\left(x^{\prime}\right)} \frac{K_{j l}^{(k)}\left(x^{\prime}, \xi^{\prime}\right)}{P_{k}\left(x^{\prime}, \xi^{\prime}\right)}\right) \\
+\int_{S} \frac{1}{2 \pi\left|x^{\prime}-\xi^{\prime}\right|^{2}} \frac{d x^{\prime}}{\cos \left(\nu_{x}, x_{3}\right)} \sum_{k=1}^{2}(-1)^{k} \sum_{j=1}^{3}\left[\beta_{i j}^{(k)}\left(\xi^{\prime}\right)-\beta_{i j}^{(k)}\left(x^{\prime}\right)\right] \\
\times\left(\left.\frac{\partial u(x)}{\partial x_{m}}\right|_{x_{3}=\gamma_{k}\left(x^{\prime}\right)} \frac{K_{j m}^{(k)}\left(x^{\prime}, \xi^{\prime}\right)}{P_{k}\left(x^{\prime}, \xi^{\prime}\right)}+\left.\frac{\partial u(x)}{\partial x_{l}}\right|_{x_{3}=\gamma_{k}\left(x^{\prime}\right)} \frac{K_{j l}^{(k)}\left(x^{\prime}, \xi^{\prime}\right)}{P_{k}\left(x^{\prime}, \xi^{\prime}\right)}\right)+\ldots
\end{gathered}
$$

The second integral in the right-hand side of (4.3) has a week singularity under the condition that the functions $\beta_{i j}^{(k)}\left(\xi^{\prime}\right)$ satisfy Hölder's condition. Before grouping the derivatives in the first integral, we expand all the coefficients at the derivatives by Taylor's formula at the point $\xi^{\prime}=x^{\prime}$ :

$$
\begin{aligned}
\frac{K_{i j}^{(k)}\left(x^{\prime}, \xi^{\prime}\right)}{P_{k}\left(x^{\prime}, \xi^{\prime}\right)} & =\frac{K_{i j}^{(k)}\left(x^{\prime}, x^{\prime}\right)}{P_{k}\left(x^{\prime}, x^{\prime}\right)}+\frac{\partial}{\partial x_{1}}\left(\frac{K_{i j}^{(k)}\left(x^{\prime}, x^{\prime}\right)}{P_{k}\left(x^{\prime}, x^{\prime}\right)}\right)\left(x_{1}-\xi_{1}\right) \\
+ & \frac{\partial}{\partial x_{2}}\left(\frac{K_{i j}^{(k)}\left(x^{\prime}, x^{\prime}\right)}{P_{k}\left(x^{\prime}, x^{\prime}\right)}\right)\left(x_{2}-\xi_{2}\right)+\ldots .
\end{aligned}
$$

All the terms except the first one reduce the order of singularity and make it weaker for a double integral over the surface S. For this reason we will consider only the first term of each expansion: 


$$
\begin{gathered}
\sum_{j=1}^{3}\left(\left.\beta_{i j}^{(1)}\left(\xi^{\prime}\right) \frac{\partial u(\xi)}{\partial \xi_{j}}\right|_{\xi_{3}=\gamma_{1}\left(\xi^{\prime}\right)}+\left.\beta_{i j}^{(2)}\left(\xi^{\prime}\right) \frac{\partial u(\xi)}{\partial \xi_{j}}\right|_{\xi_{3}=\gamma_{2}\left(\xi^{\prime}\right)}\right) \\
=\int_{S} \frac{1}{2 \pi\left|x^{\prime}-\xi^{\prime}\right|^{2}} \frac{d x^{\prime}}{\cos \left(\nu_{x}, x_{3}\right)} \sum_{k=1}^{2}(-1)^{k} \sum_{j=1}^{3} \beta_{i j}^{(k)}\left(x^{\prime}\right) \\
\times\left(\left.\frac{\partial u(x)}{\partial x_{m}}\right|_{x_{3}=\gamma_{k}\left(x^{\prime}\right)} \frac{K_{j m}^{(k)}\left(x^{\prime}, \xi^{\prime}\right)}{P_{k}\left(x^{\prime}, \xi^{\prime}\right)}+\left.\frac{\partial u(x)}{\partial x_{l}}\right|_{x_{3}=\gamma_{k}\left(x^{\prime}\right)} \frac{K_{j l}^{(k)}\left(x^{\prime}, \xi^{\prime}\right)}{P_{k}\left(x^{\prime}, \xi^{\prime}\right)}\right)+\ldots \\
=\int_{S} \frac{1}{2 \pi\left|x^{\prime}-\xi^{\prime}\right|^{2}} \frac{d x^{\prime}}{\cos \left(\nu_{x}, x_{3}\right)} \sum_{k=1}^{2}(-1)^{k} \sum_{j=1}^{3} \frac{\partial u(x)}{\partial x_{j}} \\
\times\left.\right|_{x_{3}=\gamma_{k}\left(x^{\prime}\right)}\left(\beta_{i l}^{(k)}\left(x^{\prime}\right) \frac{K_{l j}^{(k)}\left(x^{\prime}, x^{\prime}\right)}{P_{k}\left(x^{\prime}, x^{\prime}\right)}+\beta_{i m}^{(k)}\left(x^{\prime}\right) \frac{K_{m j}^{(k)}\left(x^{\prime}, x^{\prime}\right)}{P_{k}\left(x^{\prime}, x^{\prime}\right)}\right)+\ldots,
\end{gathered}
$$

where the triple $j, l, m$ is a permutation of numbers $1,2,3 ; i=1,2$.

To regularize the integral in the right-hand side of (4.4), we should impose conditions on the coefficients $\beta_{i j}^{(k)}\left(\xi^{\prime}\right)$, i.e., make the coefficients at the derivatives under the sign of the integral (4.4) equal to the coefficients $\alpha_{i j}^{(k)}\left(\xi^{\prime}\right)$ from boundary conditions (2.2). Then we get a system of 6 equations for each $i=1,2$ :

$$
(-1)^{k} \beta_{i l}^{(k)}\left(x^{\prime}\right) \frac{K_{l j}^{(k)}\left(x^{\prime}, x^{\prime}\right)}{P_{k}\left(x^{\prime}, x^{\prime}\right)}+(-1)^{k} \beta_{i m}^{(k)}\left(x^{\prime}\right) \frac{K_{m j}^{(k)}\left(x^{\prime}, x^{\prime}\right)}{P_{k}\left(x^{\prime}, x^{\prime}\right)}=\alpha_{i j}^{(k)}\left(x^{\prime}\right),
$$

$k=1,2 ; j=1,2,3$, where, as we have mentioned above, the triple $j, l, m$ is a permutation of numbers $1,2,3$.

Systems (4.5) can be written in the matrix form for $i=1,2$ :

$$
\left(\begin{array}{cccccc}
0 & 0 & \frac{K_{12}^{(1)}(x, x)}{P_{1}\left(x^{\prime}, x^{\prime}\right)} & 0 & \frac{K_{31}^{(1)}(x, x)}{P_{1}\left(x^{\prime}, x^{\prime}\right)} & 0 \\
0 & 0 & 0 & -\frac{K_{21}^{(2)}(x, x)}{P_{2}\left(x^{\prime}, x^{\prime}\right)} & 0 & -\frac{K_{31}^{(2)}(x, x)}{P_{2}\left(x^{\prime}, x^{\prime}\right)} \\
\frac{K_{12}^{(1)}(x, x)}{P_{1}\left(x^{\prime}, x^{\prime}\right)} & 0 & 0 & 0 & \frac{K_{32}^{(1)}(x, x)}{P_{1}\left(x^{\prime}, x^{\prime}\right)} & 0 \\
0 & -\frac{K_{12}^{(2)}(x, x)}{P_{2}\left(x^{\prime}, x^{\prime}\right)} & 0 & 0 & 0 & -\frac{K_{32}^{(2)}(x, x)}{P_{2}\left(x^{\prime}, x^{\prime}\right)} \\
\frac{K_{13}^{(1)}(x, x)}{P_{1}\left(x^{\prime}, x^{\prime}\right)} & 0 & \frac{K_{23}^{(1)}(x, x)}{P_{1}\left(x^{\prime}, x^{\prime}\right)} & 0 & 0 & 0 \\
0 & -\frac{K_{13}^{(2)}(x, x)}{P_{2}\left(x^{\prime}, x^{\prime}\right)} & 0 & -\frac{K_{23}^{(2)}(x, x)}{P_{2}\left(x^{\prime}, x^{\prime}\right)} & 0 & 0
\end{array}\right)
$$




$$
\times\left(\begin{array}{c}
\beta_{i 1}^{(1)}\left(x^{\prime}\right) \\
\beta_{i 1}^{(2)}\left(x^{\prime}\right) \\
\beta_{i 2}^{(1)}\left(x^{\prime}\right) \\
\beta_{i 2}^{(2)}\left(x^{\prime}\right) \\
\beta_{i 3}^{(1)}\left(x^{\prime}\right) \\
\beta_{i 3}^{(2)}\left(x^{\prime}\right)
\end{array}\right)=\left(\begin{array}{c}
\alpha_{i 1}^{(1)}\left(x^{\prime}\right) \\
\alpha_{i 1}^{(2)}\left(x^{\prime}\right) \\
\alpha_{i 2}^{(1)}\left(x^{\prime}\right) \\
\alpha_{i 2}^{(2)}\left(x^{\prime}\right) \\
\alpha_{i 3}^{(1)}\left(x^{\prime}\right) \\
\alpha_{i 3}^{(2)}\left(x^{\prime}\right)
\end{array}\right) .
$$

We reduce systems (4.6) to the triangular form:

$$
\begin{gathered}
\left(\begin{array}{cccccc}
1 & 0 & 0 & 0 & \frac{K_{32}^{(1)}}{K_{12}^{(1)}} & 0 \\
0 & 1 & 0 & 0 & 0 & \frac{K_{31}^{(2)}}{K_{12}^{(2)}} \\
0 & 0 & 1 & 0 & \frac{K_{31}^{(1)}}{K_{21}^{(1)}} & 0 \\
0 & 0 & 0 & 1 & 0 & \frac{K_{31}^{(2)}}{K_{21}^{(2)}} \\
0 & 0 & 0 & 0 & -\frac{K_{32}^{(1)}}{K_{12}^{(1)}} \frac{K_{13}^{(1)}}{P_{1}}-\frac{K_{32}^{(1)}}{P_{1}} & 0 \\
0 & \frac{K_{32}^{(2)}}{K_{12}^{(2)}} \frac{K_{13}^{(2)}}{P_{2}}+\frac{K_{23}^{(2)}}{P_{2}} \frac{K_{31}^{(2)}}{K_{21}^{(2)}}
\end{array}\right) \\
0 \\
0
\end{gathered}
$$

We suppose that systems (4.6), or (4.7), have the solutions $\left(\beta_{i 1}^{(1)}, \beta_{i 1}^{(2)}, \beta_{i 2}^{(1)}, \beta_{i 2}^{(2)}, \beta_{i 3}^{(1)}, \beta_{i 3}^{(2)}\right)$ for $i=1,2$, respectively. 
$\mathrm{R}$ e $\mathrm{m}$ a $\mathrm{r} \mathrm{k}$ 4.1. The obtain function $\beta_{i j}^{(k)}, i, k=1,2 ; j=1,2,3$, are linear functions of the given functions $\alpha_{i j}^{(k)}, i, k=1,2 ; j=1,2,3$, and, therefore satisfy Hölder condition.

Then for further regularization, we replace the expression under the integral sign in the right-hand side of (4.4) by using boundary conditions (2.2):

$$
\begin{gathered}
\sum_{j=1}^{3}\left(\left.\beta_{i j}^{(1)}\left(\xi^{\prime}\right) \frac{\partial u(\xi)}{\partial \xi_{j}}\right|_{\xi_{3}=\gamma_{1}\left(\xi^{\prime}\right)}+\left.\beta_{i j}^{(2)}\left(\xi^{\prime}\right) \frac{\partial u(\xi)}{\partial \xi_{j}}\right|_{\xi_{3}=\gamma_{2}\left(\xi^{\prime}\right)}\right) \\
=\int_{S} \frac{f_{i}\left(x^{\prime}\right)}{2 \pi\left|x^{\prime}-\xi^{\prime}\right|^{2}} \frac{d x^{\prime}}{\cos \left(\nu_{x}, x_{3}\right)} \\
-\int_{S} \frac{1}{2 \pi\left|x^{\prime}-\xi^{\prime}\right|^{2}}\left[\sum_{k=1}^{2} \alpha_{i}^{(k)}\left(x^{\prime}\right) u\left(x^{\prime}, \gamma_{k}\left(x^{\prime}\right)\right)\right] \frac{d x^{\prime}}{\cos \left(\nu_{x}, x_{3}\right)} \ldots .
\end{gathered}
$$

From necessary condition (3.2) for $u(\xi)$ on $\Gamma_{k}, k=1,2$, by discarding the term with normal derivative $\frac{\partial u}{\partial \nu_{x}}$ in the integrand and leaving only weakly singular terms, we get:

$$
\left.u(\xi)\right|_{\xi_{3}=\gamma_{k}\left(\xi^{\prime}\right)}=-\int_{S} \frac{\left.u(x)\right|_{x_{3}=\gamma_{k}\left(x^{\prime}\right)}}{2 \pi\left|x^{\prime}-\xi^{\prime}\right|^{2}} \frac{\cos \left(x-\xi, \nu_{x}\right)}{P_{k}\left(x^{\prime}, \xi^{\prime}\right)} \begin{aligned}
& \xi_{3}=\gamma_{k}\left(\xi^{\prime}\right) \\
& x_{3}\left(x^{\prime}\right)
\end{aligned} \frac{d x^{\prime}}{\cos \left(\nu_{x}, x_{3}\right)}+\ldots .
$$

Substituting necessary conditions (4.9) into (4.8), we obtain:

$$
\begin{gathered}
\sum_{j=1}^{3}\left(\left.\beta_{i j}^{(1)}\left(\xi^{\prime}\right) \frac{\partial u(\xi)}{\partial \xi_{j}}\right|_{\xi_{3}=\gamma_{1}\left(\xi^{\prime}\right)}+\left.\beta_{i j}^{(2)}\left(\xi^{\prime}\right) \frac{\partial u(\xi)}{\partial \xi_{j}}\right|_{\xi_{3}=\gamma_{2}\left(\xi^{\prime}\right)}\right) \\
=-\int_{S} \frac{1}{2 \pi\left|x^{\prime}-\xi^{\prime}\right|^{2}} \frac{d x^{\prime}}{\cos \left(\nu_{x}, x_{3}\right)} \sum_{k=1}^{2} \alpha_{i}^{(k)}\left(x^{\prime}\right) \\
\times \int_{S} \frac{\left.u(\zeta)\right|_{\zeta_{3}=\gamma_{k}\left(\zeta^{\prime}\right)} \frac{\cos \left(\zeta-\xi, \nu_{\zeta}\right)}{2 \pi\left|x^{\prime}-\zeta^{\prime}\right|^{2}} \begin{array}{l}
\zeta_{3}=\gamma_{k}\left(\zeta^{\prime}\right) \\
x_{3}=\gamma_{k}\left(x^{\prime}\right)
\end{array}}{P_{k}\left(x^{\prime}, \zeta^{\prime}\right)} \frac{d \zeta^{\prime}}{\cos \left(\nu_{\zeta}, \zeta_{3}\right)}
\end{gathered}
$$




$$
+\int_{S} \frac{f_{i}\left(x^{\prime}\right)}{2 \pi\left|x^{\prime}-\xi^{\prime}\right|^{2}} \frac{d x^{\prime}}{\cos \left(\nu_{x}, x_{3}\right)}+\ldots
$$

But it is evident that if we change the order of integration in the first integral on the right in (4.10), then the singularity vanishes (in Cauchy's sense), i.e., we obtain the regularized relationships:

$$
\begin{gathered}
\sum_{j=1}^{3}\left(\left.\beta_{i j}^{(1)}\left(\xi^{\prime}\right) \frac{\partial u(\xi)}{\partial \xi_{j}}\right|_{\xi_{3}=\gamma_{1}\left(\xi^{\prime}\right)}+\left.\beta_{i j}^{(2)}\left(\xi^{\prime}\right) \frac{\partial u(\xi)}{\partial \xi_{j}}\right|_{\xi_{3}=\gamma_{2}\left(\xi^{\prime}\right)}\right) \\
=-\sum_{k=1} \int_{S} \frac{\left.u(\zeta)\right|_{\zeta_{3}=\gamma_{k}\left(\zeta^{\prime}\right)} d \zeta^{\prime}}{\cos \left(\nu_{\zeta}, \zeta_{3}\right)} \int_{S} \alpha_{i}^{(k)}\left(x^{\prime}\right) \frac{\cos \left(\zeta-\xi, \nu_{\zeta}\right) \mid \begin{array}{l}
\zeta_{3}=\gamma_{k}\left(\zeta^{\prime}\right) \\
x_{3}=\gamma_{k}\left(x^{\prime}\right)
\end{array}}{2 \pi\left|x^{\prime}-\zeta^{\prime}\right|^{2} P_{k}\left(x^{\prime}, \zeta^{\prime}\right)} \frac{d x^{\prime}}{\cos \left(\nu_{x}, x_{3}\right)} \\
+\int_{S} \frac{f_{i}\left(x^{\prime}\right)}{2 \pi\left|x^{\prime}-\xi^{\prime}\right|^{2}} \frac{d x^{\prime}}{\cos \left(\nu_{x}, x_{3}\right)}+\ldots
\end{gathered}
$$

Thus we have established the following

Theorem 4.1. Let the conditions of Theorem 3.1 hold true. If system (4.6) is uniquely solvable, conditions (2.2) are linearly independent, the coefficients $\alpha_{i j}^{(k)}\left(x^{\prime}\right)$ for $i=1,2 ; j=\overline{1,3} ; k=1,2$, belong to some Hölder class and the remaining coefficients and kernels are continuous functions, the functions $f_{i}\left(x^{\prime}\right), i=1,2$, are continuously differentiable and vanish on the boundary $\partial S=\bar{S} \backslash S$, then the relationships (4.11) are regular.

\section{Fredholm Property of the Problem}

It is well known that

$$
\frac{\partial}{\partial x_{p}} u\left(x_{1}, x_{2}, \gamma_{k}\left(x_{1}, x_{2}\right)\right)=\left.\frac{\partial u(x)}{\partial x_{p}}\right|_{x_{3}=\gamma_{k}\left(x^{\prime}\right)}+\left.\frac{\partial u(x)}{\partial x_{3}}\right|_{x_{3}=\gamma_{k}\left(x^{\prime}\right)} \frac{\partial \gamma_{k}\left(x^{\prime}\right)}{\partial x_{p}},
$$

$k=1,2 ; p=1,2$, whence we have

$$
\left.\frac{\partial u(x)}{\partial x_{p}}\right|_{x_{3}=\gamma_{k}\left(x^{\prime}\right)}=\frac{\partial u\left(x^{\prime}, \gamma_{k}\left(x^{\prime}\right)\right)}{\partial x_{p}}-\left.\frac{\partial u(x)}{\partial x_{3}}\right|_{x_{3}=\gamma_{k}\left(x^{\prime}\right)} \frac{\partial \gamma_{k}\left(x^{\prime}\right)}{\partial x_{p}},
$$


$p=1,2 ; k=1,2$. So, the derivatives $\left.\frac{\partial u(x)}{\partial x_{1}}\right|_{x_{3}=\gamma_{k}\left(x^{\prime}\right)}$ and $\left.\frac{\partial u(x)}{\partial x_{2}}\right|_{x_{3}=\gamma_{k}\left(x^{\prime}\right)}$ are defined

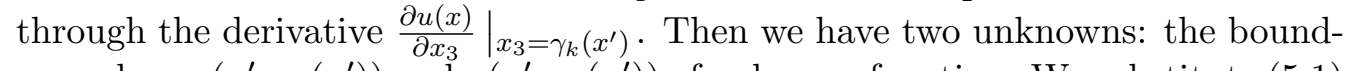
ary values $u\left(x^{\prime}, \gamma_{1}\left(x^{\prime}\right)\right)$ and $u\left(x^{\prime}, \gamma_{2}\left(x^{\prime}\right)\right)$ of unknown function. We substitute (5.1) for $\left.\frac{\partial u(x)}{\partial x_{1}}\right|_{x_{3}=\gamma_{k}\left(x^{\prime}\right)}$ and $\left.\frac{\partial u(x)}{\partial x_{2}}\right|_{x_{3}=\gamma_{k}\left(x^{\prime}\right)}$ into boundary conditions (2.2):

$$
\begin{gathered}
l_{i} u=\sum_{j=1}^{3} \sum_{m=1}^{2} \alpha_{i j}^{(m)}\left(x^{\prime}\right)\left(\frac{\partial u\left(x^{\prime}, \gamma_{m}\left(x^{\prime}\right)\right)}{\partial x_{j}}-\left.\frac{\partial u(x)}{\partial x_{3}}\right|_{x_{3}=\gamma_{m}\left(x^{\prime}\right)} \frac{\partial \gamma_{m}\left(x^{\prime}\right)}{\partial x_{j}}\right) \\
+\alpha_{i}^{(1)}\left(x^{\prime}\right) u\left(x^{\prime}, \gamma_{1}\left(x^{\prime}\right)\right)+\alpha_{i}^{(2)}\left(x^{\prime}\right) u\left(x^{\prime}, \gamma_{2}\left(x^{\prime}\right)\right)=f_{i}\left(x^{\prime}\right), \\
x^{\prime} \in S, i=1,2 .
\end{gathered}
$$

We group the unknowns to get the system

$$
\begin{aligned}
& l_{i} u=\sum_{j=1}^{2} {\left[\sum _ { m = 1 } ^ { 2 } \left[\alpha_{i j}^{(m)}\left(x^{\prime}\right)\left(\frac{\partial u\left(x^{\prime}, \gamma_{m}\left(x^{\prime}\right)\right)}{\partial x_{j}}-\left.\frac{\partial u(x)}{\partial x_{3}}\right|_{x_{3}=\gamma_{m}\left(x^{\prime}\right)} \frac{\partial \gamma_{m}\left(x^{\prime}\right)}{\partial x_{j}}\right)\right.\right.} \\
&\left.+\left.\alpha_{i 3}^{(m)}\left(x^{\prime}\right) \frac{\partial u(x)}{\partial x_{3}}\right|_{x_{3}=\gamma_{m}\left(x^{\prime}\right)}+\alpha_{i}^{(m)}\left(x^{\prime}\right) u\left(x^{\prime}, \gamma_{m}\left(x^{\prime}\right)\right)\right] \\
&-\left.\sum_{k=1}^{2} \frac{\partial u(x)}{\partial x_{3}}\right|_{x_{3}=\gamma_{k}\left(x^{\prime}\right)}\left[\sum_{m=1}^{2} \alpha_{i m}^{(k)}\left(x^{\prime}\right) \frac{\partial \gamma_{k}\left(x^{\prime}\right)}{\partial x_{m}}-\alpha_{i 3}^{(k)}\left(x^{\prime}\right)\right] \\
&+\sum_{j=1}^{2}\left[\alpha_{i j}^{(1)}\left(x^{\prime}\right) \frac{\partial u\left(x^{\prime}, \gamma_{1}\left(x^{\prime}\right)\right)}{\partial x_{j}}+\alpha_{i j}^{(2)}\left(x^{\prime}\right) \frac{\partial u\left(x^{\prime}, \gamma_{2}\left(x^{\prime}\right)\right)}{\partial x_{j}}\right] \\
&+\alpha_{i}^{(1)}\left(x^{\prime}\right) u\left(x^{\prime}, \gamma_{1}\left(x^{\prime}\right)\right)+\alpha_{i}^{(2)}\left(x^{\prime}\right) u\left(x^{\prime}, \gamma_{2}\left(x^{\prime}\right)\right)=f_{i}\left(x^{\prime}\right), \\
& x^{\prime} \in S, i=1,2 .
\end{aligned}
$$

Let us introduce the designations:

$$
A_{i j}\left(x^{\prime}\right)=-\left[\sum_{m=1}^{2} \alpha_{i m}^{(1)}\left(x^{\prime}\right) \frac{\partial \gamma_{1}\left(x^{\prime}\right)}{\partial x_{m}}-\alpha_{i 3}^{(1)}\left(x^{\prime}\right)\right], i, j=1,2 .
$$

Then system (5.2) can be written in the form

$$
\left.A_{i 1}\left(x^{\prime}\right) \frac{\partial u(x)}{\partial x_{3}}\right|_{x_{3}=\gamma_{1}\left(x^{\prime}\right)}+\left.A_{i 1}\left(x^{\prime}\right) \frac{\partial u(x)}{\partial x_{3}}\right|_{x_{3}=\gamma_{2}\left(x^{\prime}\right)}=F_{i}\left(x^{\prime}\right), i=1,2,
$$

where the right-hand sides of system (4.11) have the form:

$$
\mathrm{F}_{\mathrm{i}}\left(x^{\prime}\right)=f_{i}\left(x^{\prime}\right)-\sum_{j=1}^{2} \sum_{m=1}^{2} \alpha_{i j}^{(m)}\left(x^{\prime}\right) \frac{\partial u\left(x^{\prime}, \gamma_{m}\left(x^{\prime}\right)\right)}{\partial x_{j}}
$$




$$
+\sum_{k=1}^{2} \alpha_{i}^{(k)}\left(x^{\prime}\right) u\left(x^{\prime}, \gamma_{k}\left(x^{\prime}\right)\right), x^{\prime} \in S, i=1,2
$$

$\mathrm{R}$ e $\mathrm{m}$ a $\mathrm{rk}$ 5.1. Note that the right sides $\mathrm{F}_{\mathrm{i}}\left(x^{\prime}\right)$ of system (5.3) are functionals of the boundary values of the desired function and partial derivatives of these boundary values what follow from (5.4):

$$
\mathrm{F}_{\mathrm{i}}\left(x^{\prime}\right)=\mathrm{F}_{\mathrm{i}}\left(x^{\prime},\left.u\right|_{\Gamma_{1}},\left.u\right|_{\Gamma_{2}}, \frac{\left.\partial u\right|_{\Gamma_{1}}}{\partial x_{1}}, \frac{\left.\partial u\right|_{\Gamma_{1}}}{\partial x_{2}}, \frac{\left.\partial u\right|_{\Gamma_{2}}}{\partial x_{1}}, \frac{\left.\partial u\right|_{\Gamma_{2}}}{\partial x_{2}}\right), i=1,2 .
$$

We will reduce system (5.3) to a normal form. For this purpose the determinant of the system is required to be nonzero:

$$
\Delta\left(x^{\prime}\right)=\left|\begin{array}{ll}
A_{11}\left(x^{\prime}\right) & A_{12}\left(x^{\prime}\right) \\
A_{21}\left(x^{\prime}\right) & A_{22}\left(x^{\prime}\right)
\end{array}\right| \neq 0 .
$$

If the coefficients $\alpha_{i j}^{(k)}\left(x^{\prime}\right), i, j, k=1,2$, and the equations of the boundaries $\gamma_{1}\left(x^{\prime}\right)$ and $\gamma_{2}\left(x^{\prime}\right)$ satisfy condition (5.6), then by Cramer's formulas we have

$$
\begin{aligned}
& \left.\frac{\partial u(x)}{\partial x_{3}}\right|_{x_{3}=\gamma_{1}\left(x^{\prime}\right)}=\frac{1}{\Delta\left(x^{\prime}\right)}\left|\begin{array}{ll}
F_{1}\left(x^{\prime}\right) & A_{12}\left(x^{\prime}\right) \\
F_{2}\left(x^{\prime}\right) & A_{22}\left(x^{\prime}\right)
\end{array}\right|, \\
& \left.\frac{\partial u(x)}{\partial x_{3}}\right|_{x_{3}=\gamma_{2}\left(x^{\prime}\right)}=\frac{1}{\Delta\left(x^{\prime}\right)}\left|\begin{array}{ll}
A_{11}\left(x^{\prime}\right) & F_{1}\left(x^{\prime}\right) \\
A_{21}\left(x^{\prime}\right) & F_{2}\left(x^{\prime}\right)
\end{array}\right| .
\end{aligned}
$$

System (5.7) is a system of integro-differential Fredholm equations of the second kind with a regular kernel. Solving it, we will obtain the solution in the form:

$$
\left.\frac{\partial u(x)}{\partial x_{3}}\right|_{x_{3}=\gamma_{k}\left(x^{\prime}\right)}=\Phi_{k}\left(\left.u\right|_{\Gamma_{1}},\left.u\right|_{\Gamma_{2}}, \frac{\left.\partial u\right|_{\Gamma_{1}}}{\partial x_{1}}, \frac{\left.\partial u\right|_{\Gamma_{1}}}{\partial x_{2}}, \frac{\left.\partial u\right|_{\Gamma_{2}}}{\partial x_{1}}, \frac{\left.\partial u\right|_{\Gamma_{2}}}{\partial x_{2}}\right), k=1,2 .
$$

Going back to regular boundaries conditions (4.7), let us substitute expressions (5.1) for the derivatives $\left.\frac{\partial u(x)}{\partial x_{j}}\right|_{x_{3}=\gamma_{k}\left(x^{\prime}\right)}, j, k=1,2$, into (4.11):

$$
\begin{gathered}
\sum_{j=1}^{3}\left(\left.\beta_{i j}^{(1)}\left(\xi^{\prime}\right) \frac{\partial u(\xi)}{\partial \xi_{j}}\right|_{\xi_{3}=\gamma_{1}\left(\xi^{\prime}\right)}+\left.\beta_{i j}^{(2)}\left(\xi^{\prime}\right) \frac{\partial u(\xi)}{\partial \xi_{j}}\right|_{\xi_{3}=\gamma_{2}\left(\xi^{\prime}\right)}\right) \\
=\sum_{j=1}^{2} \sum_{m=1}^{2} \beta_{i j}^{(m)}\left(\xi^{\prime}\right)\left(\frac{\partial u\left(\xi^{\prime}, \gamma_{m}\left(\xi^{\prime}\right)\right)}{\partial \xi_{j}}-\left.\frac{\partial u(\xi)}{\partial \xi_{3}}\right|_{\xi_{3}=\gamma_{m}\left(\xi^{\prime}\right)} \frac{\partial \gamma_{m}\left(\xi^{\prime}\right)}{\partial \xi_{j}}\right) \\
+\left.\beta_{i 3}^{(1)}\left(\xi^{\prime}\right) \frac{\partial u(\xi)}{\partial \xi_{3}}\right|_{\xi_{3}=\gamma_{1}\left(\xi^{\prime}\right)}+\left.\beta_{i 3}^{(2)}\left(\xi^{\prime}\right) \frac{\partial u(\xi)}{\partial \xi_{3}}\right|_{\xi_{3}=\gamma_{2}\left(\xi^{\prime}\right)}
\end{gathered}
$$




$$
\begin{gathered}
=-\int_{S} \frac{1}{2 \pi\left|x^{\prime}-\xi^{\prime}\right|^{2}}\left[\sum_{m=1}^{2} \alpha_{i}^{(m)}\left(x^{\prime}\right) u\left(x^{\prime}, \gamma_{m}\left(x^{\prime}\right)\right)\right] \frac{d x^{\prime}}{\cos \left(\nu_{x}, x_{3}\right)} \\
\quad+\int_{S} \frac{f_{i}\left(x^{\prime}\right) d x^{\prime}}{2 \pi\left|x^{\prime}-\xi^{\prime}\right|^{2} \cos \left(\nu_{x}, x_{3}\right)}+\ldots, i=1,2 .
\end{gathered}
$$

Now we group the terms both on the left and on the right in (5.9):

$$
\begin{aligned}
& -\left.\sum_{k=1}^{2} \frac{\partial u(\xi)}{\partial \xi_{3}}\right|_{\xi_{3}=\gamma_{k}\left(\xi^{\prime}\right)}\left[\sum_{m=1}^{2} \beta_{i m}^{(k)}\left(\xi^{\prime}\right) \frac{\partial \gamma_{k}\left(\xi^{\prime}\right)}{\partial \xi_{m}}-\beta_{i 3}^{(k)}\left(\xi^{\prime}\right)\right] \\
& +\sum_{j=1}^{2}\left[\beta_{i j}^{(1)}\left(\xi^{\prime}\right) \frac{\partial u\left(\xi^{\prime}, \gamma_{1}\left(\xi^{\prime}\right)\right)}{\partial \xi_{j}}+\beta_{i j}^{(2)}\left(\xi^{\prime}\right) \frac{\partial u\left(\xi^{\prime}, \gamma_{2}\left(\xi^{\prime}\right)\right)}{\partial \xi_{j}}\right] \\
& =-\int_{S} \frac{d x^{\prime}}{2 \pi\left|x^{\prime}-\xi^{\prime}\right|^{2} \cos \left(\nu_{x}, x_{3}\right)} \sum_{m=1}^{2} \alpha_{i}^{(m)}\left(x^{\prime}\right) u\left(x^{\prime}, \gamma_{m}\left(x^{\prime}\right)\right) \\
& +\int_{S} \frac{f_{i}\left(x^{\prime}\right) d x^{\prime}}{2 \pi\left|x^{\prime}-\xi^{\prime}\right|^{2} \cos \left(\nu_{x}, x_{3}\right)}+\ldots \quad, x^{\prime} \in S, i=1,2 .
\end{aligned}
$$

The terms in (5.10) are either with weakly singular kernels or with regular ones. If we introduce the designations

$$
\begin{gathered}
C_{i j}\left(\xi^{\prime}\right)=-\left[\sum_{m=1}^{2} \beta_{i m}^{(j)}\left(\xi^{\prime}\right) \frac{\partial \gamma_{j}\left(\xi^{\prime}\right)}{\partial \xi_{m}}-\beta_{i 3}^{(j)}\left(\xi^{\prime}\right)\right], i, j=1,2, \\
B_{i}\left(\xi^{\prime}\right)=-\sum_{j=1}^{2} \sum_{m=1}^{2} \beta_{i j}^{(m)}\left(\xi^{\prime}\right) \frac{\partial u\left(\xi^{\prime}, \gamma_{m}\left(\xi^{\prime}\right)\right)}{\partial \xi_{j}} \\
-\int_{S} \frac{d x^{\prime}}{2 \pi\left|x^{\prime}-\xi^{\prime}\right|^{2} \cos \left(\nu_{x}, x_{3}\right)} \sum_{m=1}^{2} \alpha_{i}^{(m)}\left(x^{\prime}\right) u\left(x^{\prime}, \gamma_{m}\left(x^{\prime}\right)\right) \\
+\int_{S} \frac{f_{i}\left(x^{\prime}\right) d x^{\prime}}{2 \pi\left|x^{\prime}-\xi^{\prime}\right|^{2} \cos \left(\nu_{x}, x_{3}\right)}+\ldots, x^{\prime} \in S, i=1,2,
\end{gathered}
$$

then system (5.10) can be written in the form

$$
\left.\sum_{m=1}^{2} C_{i m}\left(\xi^{\prime}\right) \frac{\partial u(\xi)}{\partial \xi_{3}}\right|_{\xi_{3}=\gamma_{m}\left(\xi^{\prime}\right)}=B_{i}\left(\xi^{\prime}\right), i=1,2 .
$$


Evidently, system (5.11) is a system of integro-differential Fredholm equations of the second kind with respect to $\left.\frac{\partial u(\xi)}{\partial \xi_{3}}\right|_{\xi_{3}=\gamma_{k}\left(\xi^{\prime}\right)}, k=1,2$, and thus, has the unique solution

$$
\left.\frac{\partial u(\xi)}{\partial \xi_{3}}\right|_{\xi_{3}=\gamma_{k}\left(\xi^{\prime}\right)}=\Psi_{k}\left(\left.u\right|_{\Gamma_{1}},\left.u\right|_{\Gamma_{2}}, \frac{\left.\partial u\right|_{\Gamma_{1}}}{\partial \xi_{1}}, \frac{\left.\partial u\right|_{\Gamma_{2}}}{\partial \xi_{1}}, \frac{\left.\partial u\right|_{\Gamma_{1}}}{\partial \xi_{2}}, \frac{\left.\partial u\right|_{\Gamma_{2}}}{\partial \xi_{2}}\right),
$$

where $\left.u\right|_{\Gamma_{k}}=u\left(\xi^{\prime}, \gamma_{k}\left(\xi^{\prime}\right)\right), \frac{\left.\partial u\right|_{\Gamma_{k}}}{\partial \xi_{j}}=\frac{\partial u\left(\xi^{\prime}, \gamma_{k}\left(\xi^{\prime}\right)\right)}{\partial \xi_{j}}, j=1,2, k=1,2$, are the boundary values of the desired solution $u(x)$ on the surfaces $\Gamma_{k}, k=1,2$, and the derivatives of its boundary values, respectively.

The functionals $\Phi_{k}, \Psi_{k}, k=1,2$, from (5.8) and (5.12) are linear with respect to the unknown values $\left.u\right|_{\Gamma_{1}},\left.u\right|_{\Gamma_{2}}, \frac{\left.\partial u\right|_{\Gamma_{1}}}{\partial \xi_{j}}, \frac{\left.\partial u\right|_{\Gamma_{2}}}{\partial \xi_{j}}, j=1,2$ :

$$
\begin{gathered}
\Phi_{k}\left(\left.u\right|_{\Gamma_{1}},\left.u\right|_{\Gamma_{2}}, \frac{\left.\partial u\right|_{\Gamma_{1}}}{\partial \xi_{1}}, \frac{\left.\partial u\right|_{\Gamma_{2}}}{\partial \xi_{1}}, \frac{\left.\partial u\right|_{\Gamma_{1}}}{\partial \xi_{2}}, \frac{\left.\partial u\right|_{\Gamma_{2}}}{\partial \xi_{2}}\right) \\
=\left.\sum_{i=1}^{2} a_{i}^{(k)}\left(\xi^{\prime}\right) u\right|_{\Gamma_{i}}+\sum_{i, j=1}^{2} b_{i j}^{(k)}\left(\xi^{\prime}\right) \frac{\left.\partial u\right|_{\Gamma_{i}}}{\partial \xi_{j}}+\left.\sum_{i=1}^{2} \int_{S} c_{i}^{(k)}\left(\zeta^{\prime}\right) u\right|_{\Gamma_{i}} d \zeta \\
+\sum_{i, j=1}^{2} \int_{S} d_{i j}^{(k)}\left(\zeta^{\prime}\right) \frac{\left.\partial u\right|_{\Gamma_{i}}}{\partial \zeta_{j}} d \zeta+\varphi_{k}\left(\xi^{\prime}\right), k=1,2, \\
\Psi_{k}\left(\left.u\right|_{\Gamma_{1}},\left.u\right|_{\Gamma_{2}}, \frac{\left.\partial u\right|_{\Gamma_{1}}}{\partial \xi_{1}}, \frac{\left.\partial u\right|_{\Gamma_{2}}}{\partial \xi_{1}}, \frac{\left.\partial u\right|_{\Gamma_{1}}}{\partial \xi_{2}}, \frac{\left.\partial u\right|_{\Gamma_{2}}}{\partial \xi_{2}}\right) \\
=\left.\sum_{i=1}^{2} a_{i}^{(l)}\left(\xi^{\prime}\right) u\right|_{\Gamma_{i}}+\sum_{i, j=1}^{2} b_{i j}^{(l)}\left(\xi^{\prime}\right) \frac{\left.\partial u\right|_{\Gamma_{i}}}{\partial \xi_{j}}+\left.\sum_{i=1}^{2} \int_{S} c_{i}^{(l)}\left(\zeta^{\prime}\right) u\right|_{\Gamma_{i}} d \zeta \\
+\sum_{i, j=1}^{2} \int_{S} d_{i j}^{(l)}\left(\zeta^{\prime}\right) \frac{\left.\partial u\right|_{\Gamma_{i}}}{\partial \zeta_{j}} d \zeta+\varphi_{l}\left(\xi^{\prime}\right), l=3,4 ; k=1,2 .
\end{gathered}
$$

Excluding $\left.\frac{\partial u(\xi)}{\partial \xi_{3}}\right|_{\xi_{3}=\gamma_{k}\left(\xi^{\prime}\right)}, k=1,2$, from system (5.13), (5.14), we will obtain a system of linear integro-differential Fredholm equations of the second kind with respect to $u\left(\xi^{\prime}, \gamma_{k}\left(\xi^{\prime}\right)\right), k=1,2$ :

$$
\begin{gathered}
\left.\sum_{i=1}^{2} A_{i}^{(k)}\left(\xi^{\prime}\right) u\right|_{\Gamma_{i}}+\sum_{i, j=1}^{2} B_{i j}^{(k)}\left(\xi^{\prime}\right) \frac{\left.\partial u\right|_{\Gamma_{i}}}{\partial \xi_{j}}+\left.\sum_{i=1}^{2} \int_{S} C_{i}^{(k)}\left(\zeta^{\prime}\right) u\right|_{\Gamma_{i}} d \zeta+ \\
+\sum_{i, j=1}^{2} \int_{S} D_{i j}^{(k)}\left(\zeta^{\prime}\right) \frac{\left.\partial u\right|_{\Gamma_{i}}}{\partial \zeta_{j}} d \zeta+g_{k}\left(\xi^{\prime}\right)=0, \quad k=1,2,
\end{gathered}
$$


where

$$
\begin{gathered}
A_{i}^{(k)}\left(\xi^{\prime}\right)=a_{i}^{(k)}\left(\xi^{\prime}\right)-a_{i}^{(k+2)}\left(\xi^{\prime}\right), B_{i j}^{(k)}\left(\xi^{\prime}\right)=b_{i j}^{(k)}\left(\xi^{\prime}\right)-b_{i j}^{(k+2)}\left(\xi^{\prime}\right), \\
C_{i}^{(k)}\left(\zeta^{\prime}\right)=c_{i}^{(k)}\left(\zeta^{\prime}\right)-c_{i}^{(k+2)}\left(\zeta^{\prime}\right), D_{i j}^{(k)}\left(\zeta^{\prime}\right)=d_{i j}^{(k)}\left(\zeta^{\prime}\right)-d_{i j}^{(k+2)}\left(\zeta^{\prime}\right), \\
g_{k}\left(\xi^{\prime}\right)=\varphi_{k}\left(\xi^{\prime}\right)-\varphi_{k+2}\left(\xi^{\prime}\right), k=1,2 .
\end{gathered}
$$

Thus, we have come to a two-dimensional system of linear integro-differential equations of the first order for which Dirichlet's conditions (2.3) are given on the boundary of a two-dimensional domain S. As the boundary is one-dimensional, this Dirichlet's condition does not restrict the generality because its co-dimension is two units less than the dimension of the domain $\mathrm{D}$.

Thus, we have established the following

Theorem 5.1. Let the assumptions of Theorem 4.1 and (5.6) hold true and system (5.11) be uniquely solvable. Then boundary value problem (2.1)-(2.2) is reduced to a two-dimensional system of linear integro-differential equations (5.15) with Dirichlet's condition (2.3) on the boundary $\partial S=\bar{S} \backslash S$.

Finally, there has been established

Theorem 5.2. Under the assumptions of Theorem 5.1 boundary value problem (2.1), (2.2), (2.3) possesses the Fredholm property.

\section{References}

[1] N.A. Aliyev and A.A. Mehtiyev, Investigation of the Solutions of Boundary Value Problem for Cauchy-Riemann Type Equation in Confined Plane Field. - Journal Scientific News of Sumgayit State University 4 (2002), 30-34.

[2] N.A. Aliyev and A.Kh. Abbasova, A New Approach to the Boundary Problems for Cauchy-Riemann Equation. - Journal News of Baku State University, Phys.-Math. Sci. Series 2 (2010), 49-56.

[3] M. Jahanshahi and N.A. Aliyev, Determining of an Analytic Function on Its Analytic Domain by Cauchy-Riemann Equation with Special Kind of Boundary Conditions. - Southeast Asian Bulletin of Mathematics 1 (2004), 33-39.

[4] N.A. Aliyev, M.H. Fatehi, and M. Jahanshahi, Analytic Solution for the CauchyRiemann Equation with Non-local Boundary Conditions in the First Semi-Quarter. - Quarterly Journal of Science Tarbiat Moallem University 1 (2010), 29-40.

[5] N.A. Aliyev and M. Jahanshahi, Sufficient Conditions for Reduction of the BVP Including a Mixed PDE with Non-local Boundary Conditions to Fredholm Integral Equations. - International Journal of Mathematical Education in Science and Technology 3 (1997), 419-425. 
[6] N.A. Aliyev and M.R. Zeynalov, Steklov Problem for the First order Equation of Elliptic Type. - Journal News of Baku State University, Phys.-Math. Sci. Series 2 (2012), 12-20.

[7] M. Sajjadmanesh, M. Jahanshahi, and N. Aliyev, Inverse Problem of the Kind of Tikhonov-Lavrentiev Including the Cauchy-Riemann Equation on a Boundary Region. Book of Abstracts, The Fourth Congress of the Turkic World Mathematical Society. Azerbaijan, Baku, 1-3 July, 2011, p. 266.

[8] N.A. Aliyev, Y.Y. Mustafayeva, and S.M. Murtuzayeva, The Influence of the Carleman Condition on the Fredholm Property of the Boundary Value Problem for Cauchy-Riemann Equation. - Proceedings of the Institute of Applied Mathematics 2 (2012), 153-162.

[9] N.A. Aliyev and M. Jahanshahi, Solution of Poisson's Equation with Global, Local and Nonlocal Boundary Conditions. - International Journal of Mathematical Education in Science and Technology 2 (2002), 241-247.

[10] R.V. Huseynov, N.A. Aliyev, and S.M. Murtuzayeva, Influence of Karleman Condition by Investigating Boundary Value Problems for Laplace Equation. - Trans. Natl. Acad. Sci. Azerb., Ser. Phys.-Tech. Math. Sci. 4 (2010), 73-84.

[11] N.A. Aliev, A.Kh. Abbasova, and R.M. Zeynalov, Non-local Boundary Condition Steklov Problem for a Laplace Equation in Bounded Domain. - Science Journal of Applied Mathematics and Statistics 1 (2013), 1-6.

[12] N.A. Aliev and S.M. Hosseini, An Analysis of a Parabolic Problem with a General (Non-local and Global) Supplementary Linear Conditions. II. - Italian Journal of Pure and Applied Mathematics 13 (2003), 115-127.

[13] N.A. Aliev and S.M. Hosseini, An Analysis of a Parabolic Problem with a General (Non-local and Global) Supplementary Linear Conditions. I. - Italian Journal of Pure and Applied Mathematics 12 (2002), 143-153.

[14] N.A. Aliev and R.M. Aliguliyev, Boundary Value Problem for Equations of Hyperbolic Type. College of science works "Spectral theory of differential operators" Baku, 1984, 3-9.

[15] F.Bahrami, N.A. Aliev and S.M. Hosseini, A Method for the Reduction of Four Dimensional Mixed Problems with General Boundary Conditions to a System of Second Kind Fredholm Integral Equations. - Italian Journal of Pure and Applied Mathematics 17 (2005) 91-104.

[16] M.R. Fatemi and N.A. Aliyev, General Linear Boundary Value Problem for the Second-Order Integro-Differential Loaded Equation with Boundary Conditions Containing Both Nonlocal and Global Terms. - Journal Abstract and Applied Analysis 2010 (2010), Article ID 547526.

[17] A.Y. Delshad Gharehgheshlaghi and N.A. Aliyev, On Fredholm Property of Boundary Value Problems for a Composite Type Model Equation with General Boundary Conditions. - Intern. J. Computer Math. (2011), 124-135. 
[18] A.Y. Delshad Gharehgheshlaghi and N.A. Aliyev, General Boundary Value Problem for the Third Order Linear Differential Equation of Composite Type. - J. Math. Phys., Anal., Geom. 2 (2012), 119-134.

[19] M. Jahanshahi, N.A. Aliev, and S.M. Hosseini, An Analytic Method for Investigation and Solving Two-Dimensional Steady State Navier-Stokes Equations (I). Southeast Asian Bulletin of Mathematics 33 (2009), 749-763 (1075-1089).

[20] N.A. Aliev, Sh. Rezapour, and M.Jahanshahi, On a Mixed Problem for NavierStokes System in the Unit Cube. - Journal Mathematica Moravica 1 (2009), 13-24.

[21] N.A. Aliev and A.S. Guliev, A Boundary Value Problem for the Laplace Equation in a Three-dimensional Space. — J. Proc. Azerb. Acad. Sci., Ser. Phys.-Tech. Math. Sci. 5 (1985), 53-56.

[22] A.N. Kolmogorov and S.V. Fomin, Introductory Real Analysis. Dover Publications, New York, 1975

[23] F.G. Trikomi, Integral Equations. Interscience Publishers, New York, 1957.

[24] N.A. Aliyev and S.M. Hosseini, Multidimensional Singular Fredholm Integral Equations in a Finite Domain and Their Regularization. - Southeast Asian Bulletin of Mathematics 3 (2003), 395-408.

[25] V.S. Vladimirov, Equations of Mathematical Physics. Mir, Moscow, 1981. 